\title{
Is diabetes mellitus associated with clinical
} outcomes in aging males treated with transurethral resection of prostate for bladder outlet obstruction: implications from Taiwan Nationwide Population-Based Cohort Study

This article was published in the following Dove Press journal:

Clinical Interventions in Aging

16 March 2017

Number of times this article has been viewed

\author{
Yu-Hsiang Lin ${ }^{1-3, *}$ \\ Chen-Pang Hou ${ }^{1,2, *}$ \\ Tien-Hsing Chen ${ }^{2,4}$ \\ Horng-Heng Juang ${ }^{5}$ \\ Phei-Lang Chang ${ }^{1,2}$ \\ Pei-Shan Yang ${ }^{1,2}$ \\ Yu-Sheng Lin 2,6 \\ Chien-Lun Chen ${ }^{1,2}$ \\ Ke-Hung Tsui ${ }^{1,2}$ \\ 'Department of Urology, Chang \\ Gung Memorial Hospital - Linkou, \\ ${ }^{2} \mathrm{School}$ of Medicine, ${ }^{3} \mathrm{Graduate}$ \\ Institute of Clinical Medical Sciences, \\ College of Medicine, Chang Gung \\ University, ${ }^{4}$ Division of Cardiology, \\ Department of Internal Medicine, \\ Chang Gung Memorial Hospital, \\ Keelung, ${ }^{5}$ Department of Anatomy, \\ School of Medicine, Chang Gung \\ University, Kwei-shan, Tao-Yuan, \\ ${ }^{6}$ Division of Cardiology, Department \\ of Internal Medicine, Chang Gung \\ Memorial Hospital, Chiayi, Taiwan, \\ Republic of China \\ *These authors contributed equally \\ to this work
}

Correspondence: Ke-Hung Tsui Department of Urology, Chang Gung Memorial Hospital - Linkou, 5 Fu-Shing Street, Kweishan, Taoyuan 333, Taiwan, Republic of China

Tel $+8863328 \quad 1200$ Ext 2137

Fax +88633285818

Email khtsui@yahoo.com
Purpose: We assessed the lower urinary tract symptoms (LUTSs) and clinical outcomes between diabetes mellitus (DM) patients and non-diabetic (non-DM) patients receiving transurethral resection of prostate (TUR-P).

Methods: This analysis was a retrospective cohort study using 13 years (2000-2012) of claims data from Taiwan's National Health Insurance Research Database (NHIRD). A total of 4,887 patients who had persistent LUTSs and underwent TUR-P for prostate enlargement (benign prostate enlargement [BPE]) were enrolled and divided into two groups: DM and non-DM groups. The patients' characteristics, postoperative clinical outcomes, and the medication records after TUR-P were compared. Chi-square test was used for categorical variables and independent samples $t$-test for continuous variables. Multivariable logistic regression analysis was used to compare the risk of postoperative outcomes. Finally, we estimated the medicationfree survival rate after TUR-P using Kaplan-Meier method and compared it between study groups using log-rank test.

Results: DM group patients had a higher prevalence of comorbidities. Postoperatively, the DM group had lower rates of urinary tract infection (UTI; odds ratio [OR], 0.78; $P=0.009$ ) and higher rates of urinary retention requiring catheterization $(\mathrm{OR}, 1.35 ; P=0.01)$ within 1 month after TUR-P. A higher proportion of patients with DM took anti-muscarinics (OR, 1.23; $P=0.032)$ within the first 3 months and $\alpha$-blockers (OR, 1.18; $P=0.049$ ) during 3-12 months after receiving TUR-P. Overall, the DM group patients had a worse postoperative medicationfree survival compared to that of non-DM group patients ( $95 \%$ confidence interval $[95 \% \mathrm{CI}]$, $1.14 ; P=0.005)$.

Conclusion: DM patients require higher rates of continuing medication after TUR-P, especially anti-muscarinics in 3 months postoperatively and alpha-blocker after 3 months postoperatively. DM patients also had higher incidence of urine retention after surgery. DM patients had relatively poor treatment outcomes compared to DM-free patients.

Keywords: benign prostate hyperplasia, infection, overactive bladder, prostatectomy, urinary tract infection, lower urinary tract symptoms

\section{Introduction}

Benign prostate enlargement (BPE) is a major cause of lower urinary tract symptoms (LUTSs) in the aging male. It affects $\sim 210$ million men globally. ${ }^{1}$ In the coming decades, 
the prevalence of BPE is anticipated to grow sharply in the United States because of the aging population. ${ }^{2}$ It was estimated that there will be 32.1 million men aged $60-79$ years in 2025, 15.3 million more than at $2000 .^{2}$ The management of BPE has been a great public health challenge, with billions spent annually to treat this disease. ${ }^{3,4}$ Meanwhile, because there has recently been a marked escalation in the prevalence of diabetes globally, ${ }^{5}$ the management of patients with concomitant BPE/LUTSs and diabetes mellitus (DM) is also an important issue that cannot be ignored. It is estimated that as many as $45 \%$ of patients with diabetes have diabetic cystopathy. ${ }^{6}$ The modern definition of diabetic cystopathy is that of a progressive condition accompanied by a spectrum of clinical symptoms and urodynamic findings, ranging from an overactive bladder to a poorly contractile bladder. ${ }^{7}$ It was later observed that the bladder dysfunction related to DM is linked to specific alterations regarding simultaneously the detrusor muscle, the urothelium, the peripheral innervation of the lower urinary tract, and the control by the central nervous system (CNS). Detrusor, neurological, and urothelial dysfunctions seem to overlap and contribute to the onset of diabetic cystopathy. ${ }^{6}$ It is observed that after adjusting for age, men with diabetes were 1.42 times more likely to report moderate to severe LUTSs than their nondiabetic counterparts. ${ }^{8}$ Meanwhile, $24.8 \%$ of men with DM are diagnosed with overactive bladder, ${ }^{9}$ and men with concomitant BPE and DM have a significantly lower maximum urinary flow rate than men without DM. ${ }^{10}$ Although the relationship between BPE/LUTSs and DM has been well discussed in the literature, to the best of our knowledge, very few studies have focused on the treatment results of DM patients who have undergone transurethral resection of prostate (TUR-P). The purpose of this study is to conduct a population-based nationwide study using the National Health Insurance Research Database (NHIRD) in Taiwan to investigate the relative clinical outcomes of diabetic patients and non-diabetic patients receiving TUR-P.

\section{Methods}

\section{Data source}

Taiwan NHIRD is the largest health care database in the world, and there have been $>2,000$ published research articles derived from NHIRD. ${ }^{11}$ This study utilized Longitudinal Health Insurance Database 2000 (LHID2000) that sampled 1,000,000 individuals randomly from Taiwan's year 2000 total population of 23.75 million. There is no significant difference between the original NHIRD patient cross section and that of the LHID2000. The LHID2000, stripped of personal identifying information, contains complete medical claims and registration files for 1,000,000 enrollees, who are randomly selected from all enrollees listed in the 2000 Registry of Beneficiaries ( $\mathrm{n}=23.72$ million) under Taiwan's National Health Insurance (NHI) program. The NHI program has been in existence since 1995, and Taiwan researchers have repeatedly validated the LHID2000 cohort as accurately reflecting the NHI enrollee database cross section.

\section{Study design}

This observational nationwide cohort study compares TUR-P effects in DM patients to those in normal controls with regard to medication consumptions and clinical outcomes. We selected for research patients with benign prostatic hyperplasia (BPH) (International Classification of Disease, Ninth Revision, Clinical Modification [ICD-9-CM] codes: 600.xx) who underwent TUR-P (monopolar) (Taiwan NHI reimbursement procedure codes: 79406B, 79411B, 79412B) between January 1, 2000 and December 31, 2012. This study excluded patients with prostate cancer of any stage (ICD-9-CM codes: 185. $\mathrm{xx}$ ) to eliminate this confounding factor from the outcome. Studies were undertaken with the approval by the Institutional Review Board for the Protection of Human Subjects (Chang Gung Memorial Hospital, CGMH104-7627B). All patients provided written informed consent. We assigned the first TUR-P as the index admission if patients received repeated TUR-Ps during the study period. The variable of primary interest in this study was DM. For inclusion in our analytic cohort, we defined patients as having DM if they had at least one inpatient claim admission record or two outpatient visit claims with a diagnosis of DM (ICD-9-CM codes: 250.xx) and were prescribed with any diabetics medication within 12 months before the index TUR-P admission.

\section{Comorbidities detection}

The patients' characteristics, surgical procedure, and diagnostic codes based on the ICD-9-CM were retrieved for analyses in this study as well as comorbidities, including hypertension (ICD-9-CM codes: 401.xx-405.xx), parkinsonism (ICD-9-CM codes: 332.xx), cerebrovascular accident (ICD-9-CM codes: 430.xx-437.xx), chronic obstructive pulmonary disease (ICD-9-CM codes: 491.xx, 492.xx, 496.xx), ischemic heart disease (ICD-9-CM codes: 410.xx-414.xx), and heart failure (428.xx).

\section{Outcome detection}

Urinary tract infection (UTI) was defined as admission into the hospital with a urinary tract dysfunction-related 
diagnosis (ICD-9-CM codes: 599.0x, 595.0x) or the occurrence of at least one emergency room or outpatient visit with a diagnosis of UTI plus antibiotics prescription. Urinary retention (UR) was defined as the occurrence of any of the following procedures: intermittent catheterization program (Taiwan NHI codes: 47013C), Foley catheter indwelling (Taiwan NHI codes: 47014C), or cystostomy creation (Taiwan NHI codes: 78003C). We also analyzed the patients' BPE symptom-related pharmacy medical prescription claims for $\alpha$-blockers, bethanechol, and anti-muscarinic agents. The definition of medical prescription used was medication possession ratio (MPR) $>50 \%$, which means 2 weeks prescription in each month. We defined MPR $<50 \%$ as no medical prescription.

\section{Statistics}

Patients eligible for analysis were categorized into two study groups: DM group and non-DM group. We compared the distributions of the demographic characteristics between study groups by using chi-square test for categorical variables and independent samples $t$-test for continuous variables. The risk of postoperative outcomes (ie, UTI, UR, in-hospital death, and re-surgery) between study groups was compared by multivariable logistic regression analysis with adjustment for patients' characteristics and preoperative medication status. Similar analyses were performed to compare the urological medication usage among the two study groups during different discrete time intervals after TUR-P (1-3 and 4-12 months). Finally, we estimated the medication-free survival rate after TUR-P using Kaplan-Meier method and compared it between study groups using log-rank test. To further eliminate confounding effects, the medication-free survival rate for the study groups was compared using multivariable Cox proportional hazard models with adjustment for aforementioned covariates. All data analyses were conducted using SPSS 22 (IBM SPSS Inc, Chicago, IL, USA).

\section{Results}

There were a total of 7,214 patients who received TUR-P in our database. Among them, 524 patients had known prostate cancer before TUR-P, while 1,803 patients were found to have prostate cancer during the postoperative follow-up periods. This study excluded patients with prostate cancer at any stage because the treatment of prostate cancer would alter the patients' voiding function. The remaining 4,887 patients met our inclusion criteria, of whom 4,056 (83\%) were non-DM group, while 831 (17\%) were DM group. The selected morbidities and demographic characteristics for the two cohorts are shown in Table 1. There was no significant difference regarding age at surgery, the proportion of UTI and UR within 3 months before surgery, and the amount of tissue ablation between the two groups. Comorbidities were more prevalent in the DM group than in the non-DM group regarding hypertension, cerebrovascular accident, ischemic heart disease, and heart failure. Before TUR-P, the proportion taking $\alpha$-blockers was slightly higher in the DM group than in the non-DM group ( $83.5 \%$ vs $80.5 \%, P=0.043)$, while the proportion observed taking anti-muscarinic agents was not significantly different $(13.2 \%$ vs $10.9 \%, P=0.058)$.

Table 2 revealed the adjusted odds ratios (ORs) of the DM group compared with the non-DM group in association with postoperative complications. The DM group had lower risks of UTI (OR, $0.78 ; 95 \%$ confidence interval [95\% CI], $0.65-0.94, P=0.009)$ and higher risks of UR requiring catheterization (OR, $1.35 ; 95 \% \mathrm{CI}, 1.07-1.70, P=0.010)$ within 1 month after TUR-P. However, the group comparison was not significantly different in terms of the risks of UTI/UR during the postoperative 1 month to 1 year. During the mean follow-up time of 5.8 and 5.0 years, respectively, the risk of repeated TUR-P was small and not significantly different between the two cohorts ( $5.1 \%$ vs $5.2 \%, P=0.787)$.

The proportions of urologic drug usage after TUR-P are shown in Figure 1. In the first 3 months after surgery, a higher proportion of patients with diabetes had taken anti-muscarinic

Table I Patients' characteristics in the non-DM and DM groups

\begin{tabular}{|c|c|c|c|}
\hline Variables & $\begin{array}{l}\text { Non-DM } \\
(n=4,056)\end{array}$ & $\begin{array}{l}\text { DM } \\
(n=83 I)\end{array}$ & $P$-value \\
\hline Age, years (mean $\pm S D$ ) & $72.4 \pm 8.5$ & $72.5 \pm 7.9$ & 0.796 \\
\hline UTI within 3 months, $\mathrm{n}(\%)$ & $955(23.5)$ & $198(23.8)$ & 0.862 \\
\hline UR within 3 months, $\mathrm{n}(\%)$ & $1,536(37.9)$ & $312(37.5)$ & 0.860 \\
\hline \multicolumn{4}{|l|}{ Comorbidity, n (\%) } \\
\hline Hypertension & I,615 (39.8) & $523(62.9)$ & $<0.001$ \\
\hline Parkinsonism & $72(1.8)$ & $22(2.6)$ & 0.095 \\
\hline Cerebrovascular accident & $399(9.8)$ & $161(19.4)$ & $<0.001$ \\
\hline $\begin{array}{l}\text { Chronic obstructive pulmonary } \\
\text { disease }\end{array}$ & $538(13.3)$ & $103(12.4)$ & 0.499 \\
\hline Ischemic heart disease & $612(15.1)$ & $184(22.1)$ & $<0.001$ \\
\hline Heart failure & $136(3.4)$ & $46(5.5)$ & 0.002 \\
\hline Tissue ablation, $\mathrm{n}(\%)$ & & & 0.437 \\
\hline $5-15 \mathrm{~g}$ & $2,602(64.2)$ & $517(62.2)$ & \\
\hline $15-50 \mathrm{~g}$ & $1,222(30.1)$ & $269(32.4)$ & \\
\hline$>50 \mathrm{~g}$ & $232(5.7)$ & $45(5.4)$ & \\
\hline \multicolumn{4}{|l|}{ Medication within 3 months, $\mathrm{n}(\%)$} \\
\hline Anti-muscarinics & $444(10.9)$ & $110(13.2)$ & 0.058 \\
\hline$\alpha$-blockers & $3,265(80.5)$ & $694(83.5)$ & 0.043 \\
\hline Bethanechol & $661(16.3)$ & $142(17.1)$ & 0.575 \\
\hline Follow-up years & $5.8 \pm 3.6$ & $5.0 \pm 3.4$ & $<0.001$ \\
\hline
\end{tabular}

Abbreviations: DM, diabetes mellitus; SD, standard deviation; UTI, urinary tract infection; UR, urinary retention. 
Table 2 Comparison of postoperative clinical outcomes between non-DM and DM groups

\begin{tabular}{|c|c|c|c|c|}
\hline \multirow[t]{2}{*}{ Variables } & \multirow{2}{*}{$\begin{array}{l}\text { Non-DM } \\
(n=4,056)\end{array}$} & \multirow{2}{*}{$\begin{array}{l}\text { DM } \\
(n=83 I)\end{array}$} & \multicolumn{2}{|l|}{ DM vs non-DM } \\
\hline & & & aOR (95\% CI) & $P$-value \\
\hline \multicolumn{5}{|l|}{ UTI, n (\%) } \\
\hline$<$ I month & $1,069(26.4)$ & $188(22.6)$ & $0.78(0.65-0.94)$ & 0.009 \\
\hline I month-I year & $1,018(25.1)$ & $195(23.5)$ & $0.87(0.73-1.04)$ & 0.136 \\
\hline \multicolumn{5}{|l|}{ UR, n (\%) } \\
\hline$<$ I month & $4 I I(10.1)$ & $113(13.6)$ & I.35 (I.07-I.70) & 0.010 \\
\hline I month-I year & $470(11.6)$ & $119(14.3)$ & $1.23(0.98-1.54)$ & 0.076 \\
\hline In-hospital death, n (\%) & $9(0.2)$ & $0(0.0)$ & NA & NA \\
\hline Re-TUR-P, n (\%) & $209(5.2)$ & $42(5.1)$ & $\mathrm{I} .05(0.74-1.49)$ & 0.787 \\
\hline
\end{tabular}

Notes: Adjusted with age, preoperative UTI within 3 months, preoperative urine retention within 3 months, hypertension, Parkinsonism, cerebrovascular accident, chronic obstructive pulmonary disease, ischemic heart disease, heart failure, tissue ablation, and preoperative medication use (yes or no).

Abbreviations: DM, diabetes mellitus; aOR, adjusted odds ratio; $\mathrm{Cl}$, confidence interval; UTI, urinary tract infection; UR, urinary retention; NA, not applicable; TUR-P, transurethral resection of prostate.

drugs (OR, 1.23; 95\% CI, 1.02-1.48, $P=0.032$ ). As for $\alpha$-blockers, although the two cohorts had a similar proportion of drug usage in the first postoperative 3 months, patients with diabetes had a higher proportion of taking $\alpha$-blockers during 4-12 months after receiving TUR-P (OR, 1.18; 95\% CI, 1.01-1.38, $P=0.049$ ). The bethanechol use proportions of the two groups were similar during the two different time intervals. Finally, if we merge all the urologic drugs into account, diabetic patients had a higher proportion of drug usage during both postoperative 1-3 months (OR, 1.18; 95\% CI, 1.01-1.38, $P=0.042)$ and 4-12 months (OR, 1.25; 95\% CI, 1.07-1.47, $P=0.005)$, respectively.
Since the optimal surgical result of TUR-P is considered to be postoperatively urologic medication free, the medication-free survivals were compared between the two groups. The result showed that patients in the DM group had worse medication-free survival compared to their counterparts in either unadjusted analysis ( $P$ of log-rank test $<0.001)$ or multivariable analysis (hazard ratio [HR], 1.14; 95\% CI, 1.04-1.25, $P=0.005$ ) during the overall follow-up (Figure $2 \mathrm{~A}$ and $\mathrm{B}$ ).

\section{Discussion}

The NHIRD is nationwide and composed of anonymous eligibility, enrollment information, claims for visits, procedures, and prescription medications of $>99 \%$ of the entire estimated 23 million population of Taiwan. ${ }^{12}$ This database permits one to identify a cohort based on diagnoses, health services, surgical procedures, and drug utilization. ${ }^{11}$ Our study used the LHID that contained all inpatient and outpatient medical claims of 1 million beneficiaries randomly sampled from the NHIRD during the period of January 1, 2000 to December 31, 2012. As a result, we believe that our data are reliable and the patients' characteristics are similar to that of the whole population.

More and more evidence indicates that DM is a major cause of prostatic tissue proliferation. Insulin may increase the transcription of genes involved in sex hormone metabolism influencing the prostatic hormonal milieu. Hyperglycemia increases the amount of sex hormones entering prostatic cells, thereby influencing prostate tissue growth. ${ }^{13}$ The study

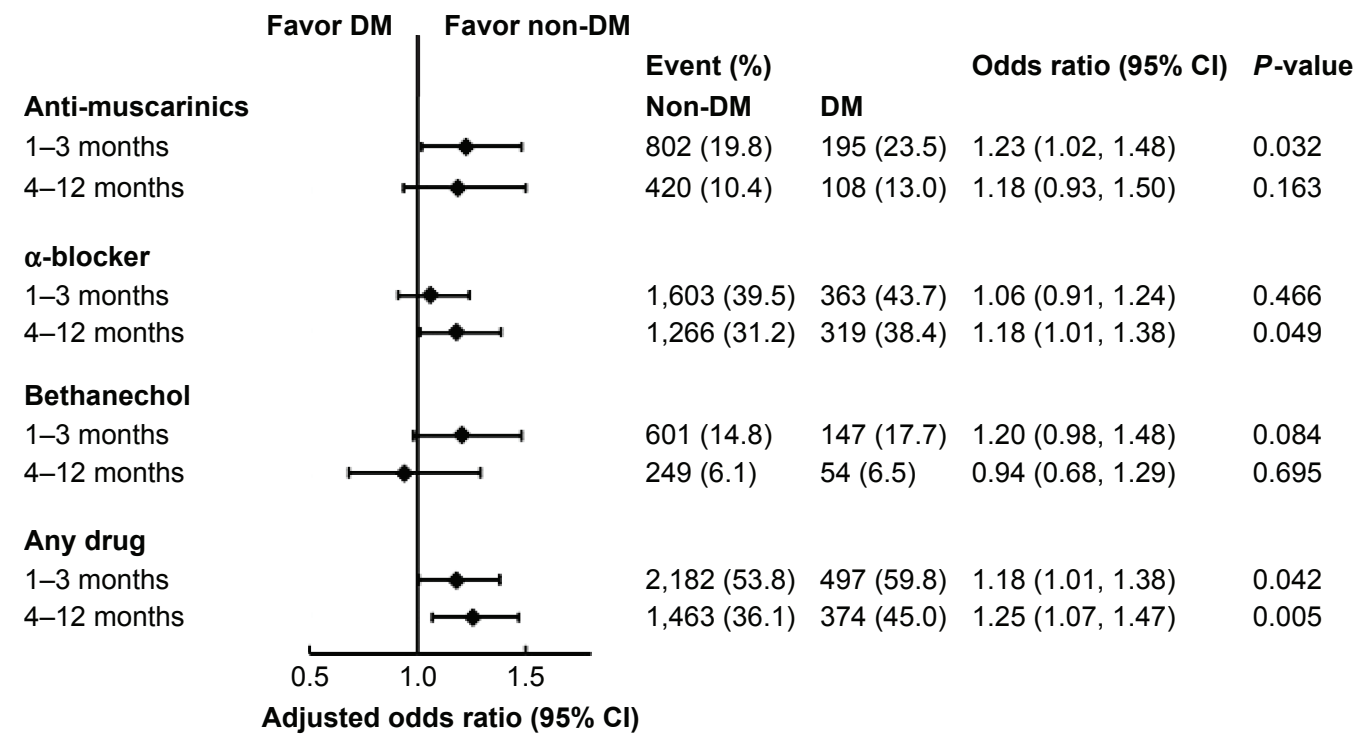

Figure I A forest plot showing the proportions of LUTS-related drug usage after TUR-P.

Abbreviations: LUTS, lower urinary tract symptom; TUR-P, transurethral resection of prostate; DM, diabetes mellitus; Cl, confidence interval. 
A

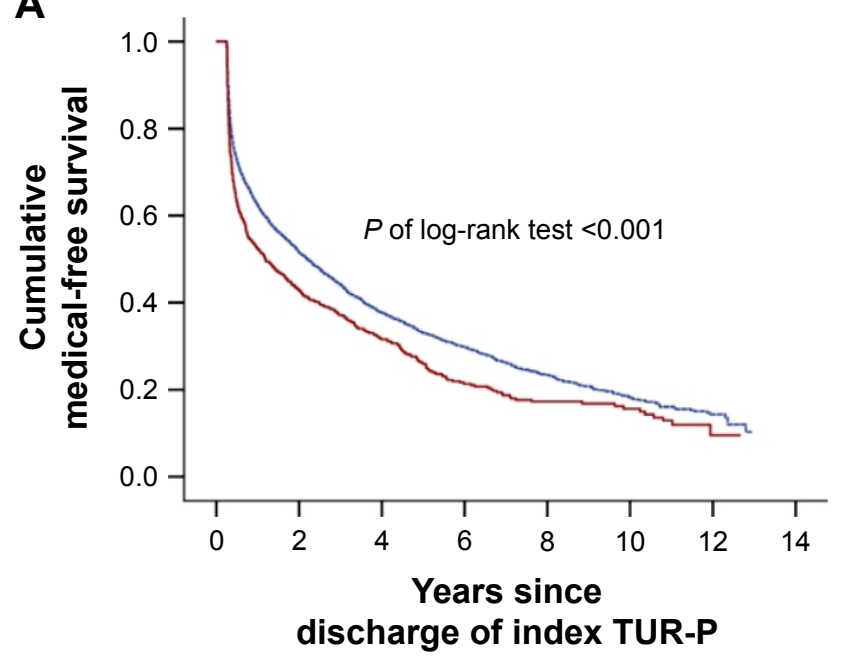

B

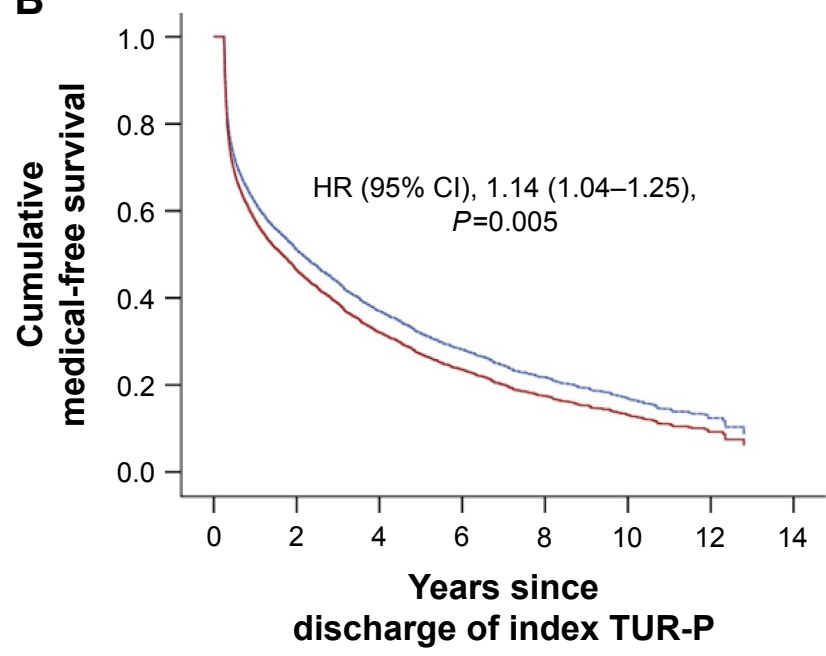

No at risk:

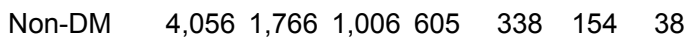

$\begin{array}{llllllll}\mathrm{DM} & 831 & 281 & 148 & 70 & 44 & 24 & 4\end{array}$
No at risk:

$\begin{array}{llllllll}\text { Non-DM } & 4,056 & 1,766 & 1,006 & 605 & 338 & 154 & 38\end{array}$

\section{Non-DM $\neg$ DM}

Figure 2 (A) Comparison of unadjusted medication-free survivals between the two groups after TUR-P. (B) Comparison of adjusted medication-free survivals between the two groups after TUR-P.

Abbreviations: TUR-P, transurethral resection of prostate; DM, diabetes mellitus; $\mathrm{HR}$, hazard ratio; $\mathrm{Cl}$, confidence interval.

of Sampson et $\mathrm{al}^{14}$ also revealed that the insulin-like growth factor (IGF) axis regulates the physiologic and pathophysiologic growth of many organs including the prostate, and dysregulation of the IGF axis has been implicated in the development of BPE. For decades, TUR-P has represented the gold standard surgical treatment of BPH with everdecreasing complication rates. ${ }^{15}$ However, very few studies have focused on the surgical results of DM patients who have undergone TUR-P.

The purpose of this study was to demonstrate the different characteristics between DM patients and non-DM patients who have undergone TUR-P. Our data reveal that DM patients who have received TUR-P have a higher incidence of other comorbidities, including hypertension, cerebrovascular accident, ischemic heart disease, and heart failure. There was no significant difference regarding age at surgery, the incidence of UTI and UR within 3 months before surgery, and the amount of tissue ablation between the two groups. However, within the first month after the patients were discharged, the DM patients had a higher incidence of UR. There were also higher percentages of DM patients who required anti-muscarinic drugs within 3 months after being discharged. In other words, compared to a non-DM patient, a patient with DM had a higher risk of being catheterized and took more time to recover from his storage symptoms in the early postoperative days. According to the study of Yamaguchi et al, ${ }^{16}$ the bladder of a DM patient is characterized by impaired bladder sensation, increased bladder capacity, and decreased contractile function of bladder. Long-standing hyperglycemia might also lead to the storage symptoms of LUTS through sympathetic nervous system activation and neuronal apoptosis. ${ }^{17}$ Furthermore, DM was the most common cause of peripheral neuropathy. The altered metabolism of glucose, tissue ischemia, superoxide-induced free-radical formation, and impaired axonal transport are all mechanisms that have been proposed to contribute to the peripheral nerve dysfunction seen in patients with DM. ${ }^{18}$ Thomas et $\mathrm{al}^{19}$ also indicated that men with the preoperative diagnosis of detrusor underactivity (DUA) had poorer surgical outcomes. Therefore, it is reasonable that a DM patient's storage symptoms would relieve more slowly and that a DM patient would experience higher rates of UR after TUR-P compared to the non-DM patients.

Our study revealed that the need of $\alpha$-blockers decreased remarkably after TUR-P on all patients. The prescription rates were similar on both groups within 3 months after being discharged. However, as time went by, the DM patients had a higher rate of $\alpha$-blocker dependence during the postoperative 4-12 months. If we further merge all the urologic drugs ( $\alpha$-blockers, anti-muscarinic agents, and bethanecol) 
into account, diabetic patients had a higher rate of drug usage during both the postoperative 1-3 months and the 4-12 months intervals. Since complete freedom from urologic medications was considered the best surgical outcome and the greatest patient wish, we used Kaplan-Meier plots to compare the medication-free survivals of the two groups to evaluate their long-term surgical results. We found that the DM group patients had a worse medication-free survival compared to the controls. In other words, the DM patients had a higher risk of continuing LUTS-associated medication, even though they had received TUR-P.

There is one interesting issue in our study that is hard to explain: the DM group had lower rates of UTI within the first month after TUR-P, which was quite different from our general concept. We believe that this result might be related to our definition of UTI. In our study, UTI was defined as admission into the hospital or emergency room or an outpatient visit with the diagnosis of UTI plus prescription of antibiotics. The urologist would possibly prescribe antibiotics if a patient said they had painful urination or bladder irritation during the first month of postoperative follow-up. It is possible that the bladder of a DM patient with cystopathy, however, is less sensitive to pain and irritation. Therefore, fewer such patients might complain to their doctors about pain symptoms, and, as a result, the percentage of UTI might be underestimated in the DM group.

This study had limitations inherited from the data structure of NHIRD. First, NHIRD did not contain personal information regarding laboratory parameters, alcohol consumption, cigarette use, and exercise that may be confounding variables influencing urinary tract symptoms. We use a propensity score to match age to minimize this potential influence. Second, the diagnoses of comorbid conditions were completely dependent on ICD-9-CM codes, especially DM, which is a key grouping factor. We adopted two strategies to overcome the defect. We adopted a DM diagnosis as at least two outpatient visits or one hospitalization that was already validated. Enrollment of DM also required prescriptions of antidiabetic medication verifiable in pharmacy claims. These methods could make study results more reliable and precise. However, we were unable to analyze the difference in outcome stratified by duration of diabetes or degree of control because we cannot obtain this information from the NHIRD database. Similarly, we cannot know exactly the proportion of our enrolled patients who truly had bladder outlet obstruction (BOO), DUA, or detrusor failure from this database. Further large-scale randomized clinical trials in the future can provide us more objective conclusions. Finally, prostatic vaporization or ablation by laser, for which there is no reimbursement in Taiwan NHI, has become more and more common in the last decades. ${ }^{20}$ Patients who received laser treatment were not included into our study cohort. Only patients receiving TUR-P are in our study population, making the study results more robust.

\section{Conclusion}

This population-based case-control study shows that patients with DM had higher rates of continuing medication after TUR-P. DM patients also had higher incidence of urine retention after surgery. In other words, they had relatively poor treatment outcomes compared to DM-free patients. Taken together, the urologist has to carefully study before surgery if DM patients had problems with LUTSs and BOO. Further large-scale prospective clinical studies and well-designed basic research are needed before definite conclusions can be drawn.

\section{Acknowledgments}

This work was supported by Chang Gung Memorial Hospital (grant numbers: CRPG3A1061, CMRPG3A1062, CMRPG3E1071) and the National Science Council, Taiwan (NSC 104-2314-B-182A-140-MY3).

\section{Disclosure}

The authors report no conflicts of interest in this work.

\section{References}

1. Verhamme KM, Dieleman JP, Bleumink GS, et al; Triumph Pan European Expert Panel. Incidence and prevalence of lower urinary tract symptoms suggestive of benign prostatic hyperplasia in primary care - the Triumph project. Eur Urol. 2002;42(4):323-328.

2. Litman HJ, McKinlay JB. The future magnitude of urologic symptoms in the USA: projections using the Boston Area Community Health survey. BJU Int. 2007;100(4):820-825.

3. Saigal CS, Joyce G. Economic costs of benign prostatic hyperplasia in the private sector. $J$ Urol. 2005;173(4):1309-1313.

4. Irwin DE, Kopp ZS, Agatep B, Milsom I, Abrams P. Worldwide prevalence estimates of lower urinary tract symptoms, overactive bladder, urinary incontinence and bladder outlet obstruction. BJU Int. 2011; 108(7):1132-1138.

5. NCD Risk Factor Collaboration (NCD-RisC). Worldwide trends in diabetes since 1980: a pooled analysis of 751 population-based studies with 4.4 million participants. Lancet. 2016;387(10027):1513-1530.

6. Sasaki K, Yoshimura N, Chancellor MB. Implications of diabetes mellitus in urology. Urol Clin North Am. 2003;30(1):1-12.

7. Yuan Z, Tang Z, He C, Tang W. Diabetic cystopathy: a review. J Diabetes. 2015;7(4):442-447.

8. Sarma AV, Burke JP, Jacobson DJ, et al. Associations between diabetes and clinical markers of benign prostatic hyperplasia among communitydwelling Black and White men. Diabetes Care. 2008;31(3):476-482.

9. Liu RT, Chung MS, Lee WC, et al. Prevalence of overactive bladder and associated risk factors in 1359 patients with type 2 diabetes. Urology. 2011;78(5):1040-1045. 
10. Michel MC, Mehlburger L, Schumacher H, Bressel HU, Goepel M. Effect of diabetes on lower urinary tract symptoms in patients with benign prostatic hyperplasia. J Urol. 2000;163(6):1725-1729.

11. Hsing AW, Ioannidis JP. Nationwide population science: lessons from the Taiwan National Health Insurance Research Database. JAMA Intern Med. 2015;175(9):1527-1529.

12. Hsiao F, Yang C, Huang Y, et al. Using Taiwan's national health insurance research databases for pharmacoepidemiology research. J Food Drug Anal. 2007;15(2):99.

13. Jangir RN, Jain GC. Diabetes mellitus induced impairment of male reproductive functions: a review. Curr Diabetes Rev. 2014;10(3):147-157.

14. Sampson N, Zenzmaier C, Heitz M, et al. Stromal insulin-like growth factor binding protein 3 (IGFBP3) is elevated in the diseased human prostate and promotes ex vivo fibroblast-tomyofibroblast differentiation. Endocrinology. 2013;154(8):2586-2599.

15. Rassweiler J, Teber D, Kuntz R, et al. Complications of transurethral resection of the prostate (TURP) - incidence, management, and prevention. Eur Urol. 2006;50(5):969-980.
16. Yamaguchi C, Sakakibara R, Uchiyama T, et al. Overactive bladder in diabetes: a peripheral or central mechanism? Neurourol Urodyn. 2007;26(6):807-813.

17. Moul S, McVary KT. Lower urinary tract symptoms, obesity and the metabolic syndrome. Curr Opin Urol. 2010;20(1):7-12.

18. Ozturk Y, Altan VM, Yildizoglu-Ari N. Effects of experimental diabetes and insulin on smooth muscle functions. Pharmacol Rev. 1996;48(1):69-112.

19. Thomas AW, Cannon A, Bartlett E, Ellis-Jones J, Abrams P. The natural history of lower urinary tract dysfunction in men: minimum 10-year urodynamic follow-up of untreated bladder outlet obstruction. BJU Int. 2005;96(9):1301-1306.

20. Lee WC, Lin YH, Hou CP, et al. Prostatectomy using different lasers for the treatment of benign prostate hyperplasia in aging males. Clin Interv Aging. 2013;8:1483-1488.
Clinical Interventions in Aging

\section{Publish your work in this journal}

Clinical Interventions in Aging is an international, peer-reviewed journal focusing on evidence-based reports on the value or lack thereof of treatments intended to prevent or delay the onset of maladaptive correlates of aging in human beings. This journal is indexed on PubMed Central, MedLine,

\section{Dovepress}

CAS, Scopus and the Elsevier Bibliographic databases. The manuscript management system is completely online and includes a very quick and fair peer-review system, which is all easy to use. Visit http://www.dovepress. com/testimonials.php to read real quotes from published authors. 